\title{
PET Imaging of PARP Expression Using ${ }^{18}$ F-Olaparib
}

\author{
Delphine L. Chen \\ Divisions of Radiological Sciences and Nuclear Medicine, Mallinckrodt Institute of Radiology, Washington University School of \\ Medicine, St. Louis, Missouri
}

See the associated article on page 504.

$\mathbf{P}$

oly(adenosine diphosphate ribose)polymerases (PARP) are an abundant family of enzymes with multiple cellular functions. PARP1, the best-studied of this family, is essential for the repair of single-strand DNA breaks through base excision repair (1). In the presence of defects in the homologous recombination DNA repair pathway (HRD), PARP1 also plays a critical role in maintaining the DNA repair machinery because the loss of PARP1 increases the number of DNA lesions normally repaired by HR (2). The breast cancer susceptibility genes (BRCA1/2) are central regulators of the HRD repair pathway $(3,4)$. Tumors with loss-of-function BRCA mutations exhibit high levels of genomic instability, thus making them dependent on PARP1 for survival. The therapeutic potential in exploiting this dependency was first illustrated in 2 key studies demonstrating that PARP inhibition in BRCA mutant cancers leads to synthetic lethality $(5,6)$. This was confirmed in initial clinical trials by marked clinical responses to PARP inhibition in patients with treatment-resistant BRCA mutant cancers $(7,8)$, sparking significant enthusiasm for this therapeutic approach. Positive results in BRCA-mutant ovarian cancer led to the Food and Drug Administration approval of olaparib (9), for which testing was required to demonstrate the presence of a BRCA mutation.

Since then, it has become clear that BRCA mutation status alone does not predict which patients will respond, as seen in an early study with olaparib, which showed objective responses in $24 \%$ of patients with ovarian cancer without a BRCA mutation (10). However, HRD markers may improve on patient selection. Niraparib and rucaparib were approved without the need for BRCA testing because phase 3 trials showed improved progression-free survival in patients either with a germline BRCA mutation or with tumors exhibiting HRD but without a germline BRCA mutation $(11,12)$. Similar results were observed in prostate cancer patients; those with genetic mutations predicting the presence of any DNA repair defect had higher response rates to olaparib (13). Olaparib has since gained expansion of the initial approved indication for maintenance therapy without needing BRCA testing (14).

Despite these advances in identifying patients who will respond, HRD markers still do not perfectly identify all responders to PARP

Received Oct. 7, 2018; revision accepted Dec. 14, 2018.

For correspondence or reprints contact: Delphine L. Chen, Washington University School of Medicine, Campus Box 8225, 510 S. Kingshighway

Blvd., St. Louis, MO 63110.

E-mail: chend@wustl.edu

Published online Jan. 10, 2019.

COPYRIGHT (c) 2019 by the Society of Nuclear Medicine and Molecular Imaging. DOI: 10.2967/jnumed.118.219733 inhibitors, nor do they predict the development of treatmentresistance mechanisms. The increase in progression-free survival in ovarian cancer patients without germline BRCA and positive HRD markers was consistently less than that seen in patients with germline BRCA in the niraparib and rucaparib trials, demonstrating the limitations of the HRD markers for predicting PARP inhibitor response. Additionally, treatment resistance can be present because of DNA replication fork stabilization, regardless of HR status, or may develop because of somatic reversion mutations that restore HR functionality during PARP inhibitor treatment (15-18). The only way to detect these alterations, whether before starting PARP inhibitor therapy or when assessing causes of treatment failure, is to obtain tumor tissue for testing. Presumably, these functional alterations affect PARP expression levels, but the study of this effect has been limited by the requirement of tissue for such assessments.

Imaging PARP expression levels may be a novel approach for studying the relationship of PARP expression and PARP inhibitor efficacy in the context of HRD and functional alterations that may confer treatment resistance. The results of such studies could be used to identify rational therapy combinations with PARP inhibitors to maximize the therapeutic benefits while minimizing exposure to toxicities in patients who would not respond. Studies have shown that PARP inhibitor efficacy in vitro requires the presence of PARP1 because PARP inhibitors are ineffective at killing PARP1 knockout cells $(19,20)$. Therefore, radiolabeled PARP inhibitors could be used to verify the presence and extent of tumoral PARP1 expression noninvasively in patients. Radiolabeled PARP inhibitors could further be used to characterize dynamic changes in tumoral PARP expression during treatment with PARP inhibitors or DNA-damaging agents that could then be related to the presence of HRD markers or other markers of treatment resistance.

Human studies performed with ${ }^{18} \mathrm{~F}$-fluorthanatrace demonstrate the potential for PARP imaging to detect differences in tumoral PARP expression in clinical studies. First-in-human studies demonstrated high ${ }^{18} \mathrm{~F}$-fluorthanatrace binding in a single-patient example of biphenotypic hepatocellular carcinoma and cholangiocarcinoma (21). A subsequent trial in ovarian cancer patients demonstrated heterogeneously increased ${ }^{18} \mathrm{~F}$-fluorthanatrace uptake that differed from ${ }^{18} \mathrm{~F}$-FDG uptake (22). This study further demonstrated that ${ }^{18} \mathrm{~F}$-fluorthanatrace uptake but not ${ }^{18} \mathrm{~F}$-FDG uptake correlated with PARP levels assessed by autoradiography and immunohistochemical staining (22). The results from these initial studies indicate that radiolabeled PARP inhibitors promise to be highly useful in measuring changes in tumoral PARP expression levels during treatment.

The relationship between PARP inhibitor binding in vivo and treatment efficacy could also be studied using radiolabeled PARP imaging agents, thus providing insights into dosing approaches for PARP inhibitor therapy. All PARP inhibitors in clinical use or under evaluation to date bind to the PARP binding site for 
oxidized nicotinamide adenine dinucleotide. The efficacy of PARP inhibitors is related to their ability to trap PARP-DNA complexes (23), and PARP inhibitors that better trap PARP-DNA complexes are more difficult to compete off with excess oxidized nicotinamide adenine dinucleotide $(19,24)$. The published radiolabeled imaging agents to date, ${ }^{18} \mathrm{~F}$-fluorthanatrace and ${ }^{18} \mathrm{~F}$-PARPi, are derived from rucaparib and olaparib, respectively, and bind to the same oxidized nicotinamide adenine dinucleotide binding site as therapeutic PARP inhibitors (25). Therefore, another compelling application for PARP imaging is to assess the ability of therapeutic PARP inhibitors to block the uptake of radiolabeled PARP imaging agents as a surrogate measure of complete target engagement during therapy. The potential of this application was initially investigated with olaparib and iniparib, a cysteine-modifying drug devoid of PARP inhibitory activity, in which olaparib but not iniparib consistently blocked the uptake of ${ }^{18} \mathrm{~F}$-fluorthanatrace in cancers and normal lymph nodes in mice $(21,25)$. Drug dosing studies to assess the potential for ${ }^{18} \mathrm{~F}$-PARP inhibitors to study intratumoral drug target engagement further demonstrated differences in the ability of olaparib and talazoparib to displace ${ }^{18} \mathrm{~F}$ PARPi relative to treatment efficacy (26). Similar studies could be performed in patients to study the kinetics of PARP inhibition during PARP inhibitor therapy to ensure consistent binding of PARP inhibitors to their target site.

In light of these potential applications, ${ }^{18} \mathrm{~F}$-olaparib promises to further the evaluation of studying PARP expression in response to PARP inhibitor and other therapies as outlined above. The investigators have developed a novel method for labeling olaparib with ${ }^{18} \mathrm{~F}$ to maintain the same chemical structure, adding another agent to the growing armamentarium of radiolabeled PARP inhibitors. ${ }^{18} \mathrm{~F}$-olaparib thus enables olaparib-specific tumoral pharmacokinetic and pharmacodynamic studies that can provide insights into the efficacy of olaparib binding in tumors at different sites. These insights would be particularly useful in studying the penetrance of olaparib into brain tumors relative to other PARP inhibitors. ${ }^{18} \mathrm{~F}$-olaparib could further be used to study the effects of PARP inhibitors on normal organs, thus providing a potential window into dose-limiting toxicities associated with olaparib treatment, particularly when used in conjunction with other myelosuppressive agents, because normal PARP expression is seen in the marrow and nodes. Finally, ${ }^{18} \mathrm{~F}$-olaparib could be used to study how olaparib binding compares with that of other approved PARP inhibitors or those in development.

In conclusion, radiolabeling of PARP inhibitors holds great promise for furthering their development as targeted anticancer therapeutics. Radiolabeled PARP inhibitors could greatly aid in studying the impact of PARP inhibitors and DNA-damaging agents on tumoral PARP expression, providing novel insights into the behavior of intratumoral PARP levels during treatment or providing drug-binding information to help predict efficacy. The potential for using information from radiolabeled PARP inhibitors to improve patient selection and outcomes for PARP inhibitor treatment, as well as to devise rational combinatorial treatment approaches with PARP inhibitors, is additional compelling justification for further developing this growing area of molecular imaging.

\section{DISCLOSURE}

Delphine Chen is a coinventor on patent WO 2015103526 A1. No other potential conflict of interest relevant to this article was reported.

\section{REFERENCES}

1. Schreiber V, Ame JC, Dolle P, et al. Poly(ADP-ribose) polymerase-2 (PARP-2) is required for efficient base excision DNA repair in association with PARP-1 and XRCC1. J Biol Chem. 2002;277:23028-23036.

2. Ashworth A. A synthetic lethal therapeutic approach: poly(ADP) ribose polymerase inhibitors for the treatment of cancers deficient in DNA double-strand break repair. J Clin Oncol. 2008;26:3785-3790.

3. Moynahan ME, Chiu JW, Koller BH, Jasin M. Brcal controls homology-directed DNA repair. Mol Cell. 1999;4:511-518.

4. Moynahan ME, Pierce AJ, Jasin M. BRCA2 is required for homology-directed repair of chromosomal breaks. Mol Cell. 2001;7:263-272.

5. Farmer H, McCabe N, Lord CJ, et al. Targeting the DNA repair defect in BRCA mutant cells as a therapeutic strategy. Nature. 2005;434:917-921.

6. Bryant HE, Schultz N, Thomas HD, et al. Specific killing of BRCA2-deficient tumours with inhibitors of poly(ADP-ribose) polymerase. Nature. 2005;434: 913-917.

7. Fong PC, Boss DS, Yap TA, et al. Inhibition of poly(ADP-ribose) polymerase in tumors from BRCA mutation carriers. N Engl J Med. 2009;361:123-134.

8. Drew Y. The development of PARP inhibitors in ovarian cancer: from bench to bedside. Br J Cancer. 2015;113(suppl 1):S3-S9.

9. Kim G, Ison G, McKee AE, et al. FDA approval summary: olaparib monotherapy in patients with deleterious germline BRCA-mutated advanced ovarian cancer treated with three or more lines of chemotherapy. Clin Cancer Res. 2015;21:4257-4261.

10. Gelmon KA, Tischkowitz M, Mackay H, et al. Olaparib in patients with recurrent high-grade serous or poorly differentiated ovarian carcinoma or triple-negative breast cancer: a phase 2, multicentre, open-label, non-randomised study. Lancet Oncol. 2011;12:852-861.

11. Mirza MR, Monk BJ, Herrstedt J, et al. Niraparib maintenance therapy in platinum-sensitive, recurrent ovarian cancer. N Engl J Med. 2016;375:2154-2164.

12. Coleman RL, Oza AM, Lorusso D, et al. Rucaparib maintenance treatment for recurrent ovarian carcinoma after response to platinum therapy (ARIEL3): a randomised, double-blind, placebo-controlled, phase 3 trial. Lancet. 2017;390: 1949-1961.

13. Mateo J, Carreira S, Sandhu S, et al. DNA-repair defects and olaparib in metastatic prostate cancer. $N$ Engl J Med. 2015;373:1697-1708.

14. Ledermann J, Harter P, Gourley C, et al. Olaparib maintenance therapy in patients with platinum-sensitive relapsed serous ovarian cancer: a preplanned retrospective analysis of outcomes by BRCA status in a randomised phase 2 trial. Lancet Oncol. 2014;15:852-861.

15. Kondrashova O, Nguyen M, Shield-Artin K, et al. Secondary somatic mutations restoring RAD51C and RAD51D associated with acquired resistance to the PARP inhibitor rucaparib in high-grade ovarian carcinoma. Cancer Discov. 2017;7:984-998.

16. Choi YE, Meghani K, Brault ME, et al. Platinum and PARP inhibitor resistance due to overexpression of microRNA-622 in BRCA1-mutant ovarian cancer. Cell Reports. 2016;14:429-439.

17. McCormick A, Donoghue P, Dixon M, et al. Ovarian cancers harbor defects in nonhomologous end joining resulting in resistance to rucaparib. Clin Cancer Res. 2017;23:2050-2060.

18. Rondinelli B, Gogola E, Yucel H, et al. EZH2 promotes degradation of stalled replication forks by recruiting MUS81 through histone H3 trimethylation. Nat Cell Biol. 2017;19:1371-1378.

19. Murai J, Huang SY, Das BB, et al. Trapping of PARP1 and PARP2 by clinical PARP inhibitors. Cancer Res. 2012;72:5588-5599.

20. Makvandi M, Xu K, Lieberman BP, et al. A radiotracer strategy to quantify PARP-1 expression in vivo provides a biomarker that can enable patient selection for PARP inhibitor therapy. Cancer Res. 2016;76:4516-4524.

21. Michel LS, Dyroff S, Brooks FJ, et al. PET of Poly (ADP-ribose) polymerase activity in cancer: preclinical assessment and first in-human studies. Radiology. 2017;282:453-463.

22. Makvandi M, Pantel A, Schwartz L, et al. A PET imaging agent for evaluating PARP-1 expression in ovarian cancer. J Clin Invest. 2018;128:2116-2126.

23. De Lorenzo SB, Patel AG, Hurley RM, Kaufmann SH. The elephant and the blind men: making sense of PARP inhibitors in homologous recombination deficient tumor cells. Front Oncol. 2013;3:228.

24. Murai J, Huang SY, Renaud A, et al. Stereospecific PARP trapping by BMN 673 and comparison with olaparib and rucaparib. Mol Cancer Ther. 2014;13:433443.

25. Zhou D, Chu W, Xu J, et al. Synthesis, $\left[{ }^{18} \mathrm{~F}\right]$ radiolabeling, and evaluation of poly (ADP-ribose) polymerase-1 (PARP-1) inhibitors for in vivo imaging of PARP-1 using positron emission tomography. Bioorg Med Chem. 2014;22:1700-1707.

26. Carney B, Kossatz S, Lok BH, et al. Target engagement imaging of PARP inhibitors in small-cell lung cancer. Nat Commun. 2018;9:176. 